\title{
Infinite approximate subgroups of soluble Lie groups
}

\author{
Simon Machado ${ }^{1}$ \\ Received: 12 November 2019 / Revised: 9 June 2021 / Accepted: 13 August 2021 / \\ Published online: 28 August 2021 \\ (c) The Author(s) 2021
}

\begin{abstract}
We study infinite approximate subgroups of soluble Lie groups. We show that approximate subgroups are close, in a sense to be defined, to genuine connected subgroups. Building upon this result we prove a structure theorem for approximate lattices in soluble Lie groups. This extends to soluble Lie groups a theorem about quasi-crystals due to Yves Meyer.
\end{abstract}

Keywords Approximate lattices · Approximate subgroups · Lie groups · Linear algebraic groups

Mathematics Subject Classification 05E15 · 22E99 · 22E40

\section{Introduction}

Approximate subgroups were defined by Terence Tao [21] in order to give a noncommutative generalisation of results from additive combinatorics. On the one hand, finite approximate subgroups have been extensively studied. We can mention, among others, Gregory Freiman [7], Harald Helfgott [9], Ehud Hrushovski [10], László Pyber and Endre Szabó [16], Imre Ruzsa [18] and Emmanuel Breuillard, Ben Green and Terence Tao [4]. This lead to the structure theorem [4] that asserts that finite approximate subgroups are commensurable to coset nilprogressions, which are a certain non-commutative generalisation of arithmetic progressions. On the other hand, it

Communicated by Andreas Thom.

This work was supported by the UK Engineering and Physical Sciences Research Council (EPSRC) grant EP/L016516/1 for the University of Cambridge Centre for Doctoral Training, the Cambridge Centre for Analysis.

$凶$ Simon Machado

sm2344@cam.ac.uk

1 Centre for Mathematical Sciences, University of Cambridge, Wilberforce Road, Cambridge CB3 0WA, UK 
seems hopeless to aim at classifying all infinite approximate subgroups. Some results in this direction for particular classes of infinite approximate subgroups can be found in $[5,10,14]$. Inspired by Yves Meyer's results on quasi-crystals [15], Michael Björklund and Tobias Hartnick have defined a class of infinite approximate subgroups called approximate lattices in [2]. These approximate subgroups generalise lattices (discrete subgroups of Lie groups with finite co-volume) and share many properties with them. For instance, lattices and approximate lattices in nilpotent Lie groups have a very similar theory, see [12]. Whether similar results hold for other types of locally compact groups is the open question that motivates this article. Here, we address the case of soluble Lie groups (Theorem 3 below). Along the way, we show a structure theorem for all approximate subgroups in soluble algebraic groups (Theorem 1).

A subset $\Lambda$ of a group $G$ is an approximate subgroup if it contains the identity, if it is symmetric i.e. $\Lambda=\Lambda^{-1}$, and if there exists a finite subset $F \subset G$ such that $\Lambda^{2} \subset F \Lambda$. Here, $\Lambda^{2}:=\left\{\lambda_{1} \lambda_{2} \mid \lambda_{1}, \lambda_{2} \in \Lambda\right\}, F \Lambda:=\{f \lambda \mid f \in F, \lambda \in \Lambda\}$ and more generally $\Lambda^{n}:=\left\{\lambda_{1} \cdots \lambda_{n} \mid \lambda_{1}, \ldots, \lambda_{n} \in \Lambda\right\}$. Moreover, set $\Lambda^{\infty}:=\bigcup_{n \geq 0} \Lambda^{n}$ the subgroup generated by $\Lambda$. We will say that two subsets $\Lambda, \Xi \subset G$ are commensurable if there is a finite set $F$ such that $\Lambda \subset F \Xi$ and $\Xi \subset F \Lambda$. If $G$ is endowed with the structure of a topological group, we say that subsets $\Lambda, \Xi \subset G$ are compactly commensurable if there is a compact subset $K \subset G$ with $\Lambda \subset K \Xi$ and $\Xi \subset K \Lambda$. Commensurability and compact commensurability are equivalence relations.

Our main result is concerned with classifying certain soluble approximate subgroups up to compact commensurability:

Theorem 1 Let $\Lambda \subset \mathrm{GL}_{n}(\mathbb{R})$ be an approximate subgroup generating a soluble subgroup. Then $\Lambda$ is compactly commensurable to a Zariski-closed soluble subgroup $H$ of $\mathrm{GL}_{n}(\mathbb{R})$ normal in the Zariski-closure of $\Lambda^{\infty}$.

Theorem 1 is a non-commutative generalisation of a theorem due to Jean-Pierre Schreiber [19, Proposition 2], which was recently given a new proof and an extension to discrete approximate subgroups of the Heisenberg group by Alexander Fish in [6, Theorem 2.2]. Theorem 1 also generalises a result of Fried and Goldman about the existence of syndetic hulls for virtually solvable subgroups of $\mathrm{GL}_{n}(\mathbb{R})$ (see $[8$, Theorem 1.6] and Proposition 2 below). Another interesting corollary to this result is that approximate lattices (see [2, Definition 4.9]) in soluble algebraic groups are uniform (see Theorem 5).

An approximate subgroup $\Lambda \subset G$ in a locally compact group is a uniform approximate lattice if $\Lambda^{2}$ is discrete and $\Lambda$ is compactly commensurable to $G$. The approximate group condition arises naturally from the combination of discreteness and compact commensurability to the ambient group: if a subset $\Lambda \subset G$ is symmetric, compactly commensurable to $G$ and $\Lambda^{6}$ is discrete, then $\Lambda$ is a uniform approximate lattice. See [2] for this and more on the general theory of approximate lattices.

Examples of uniform approximate lattices are given by cut-and-project schemes. A cut-and-project scheme $(G, H, \Gamma)$ consists of two locally compact groups $G$ and $H$, and a uniform lattice $\Gamma$ in $G \times H$ such that $\Gamma \cap\left(\left\{e_{G}\right\} \times H\right)=\left\{e_{G \times H}\right\}$ and $\Gamma$ projects densely to $H$. Given a cut-and-project scheme $(G, H, \Gamma)$ and a symmetric relatively compact neighbourhood $W_{0}$ of $e_{H}$ in $H$, one gets a uniform approximate lattice when considering the projection $\Lambda$ of $\left(G \times W_{0}\right) \cap \Gamma$ to $G$. Any approximate subgroup of $G$ 
which is commensurable to such a $\Lambda$ is called a Meyer subset of $G$. This construction was first introduced by Yves Meyer in the abelian case [15] and extended by Michael Björklund and Tobias Hartnick [2].

In a similar fashion, for $\Lambda \subset G$ an approximate subgroup, we say that a group homomorphism $f: \Lambda^{\infty} \rightarrow H$ with $H$ a locally compact group is a good model (of $\Lambda$ ) if: $(i) f(\Lambda)$ is relatively compact, and (ii) there is $V$ a neighbourhood of the identity in $H$ such that $f^{-1}(V) \subset \Lambda$. In this situation, we say that $\Lambda$ has a good model.

If $\Lambda:=p_{G}\left(G \times W_{0} \cap \Gamma\right)$ is a uniform approximate lattice constructed from a cut-and-project scheme $(G, H, \Gamma)$, then $f=p_{H} \circ\left(p_{G}\right)_{\mid \Gamma}^{-1}$ is a good model of $\Lambda$, where $p_{G}$ and $p_{H}$ are the natural projections on $G$ and $H$ respectively. Conversely, if $\Lambda \subset G$ is a uniform approximate lattice and has a good model $f$, the map

$$
\begin{aligned}
\Lambda^{\infty} & \rightarrow G \times \overline{f\left(\Lambda^{\infty}\right)} \\
\gamma & \mapsto(\gamma, f(\gamma))
\end{aligned}
$$

embeds $\Lambda^{\infty}$ in $G \times \overline{f\left(\Lambda^{\infty}\right)}$ as a uniform lattice. Thus, $\left(G, \overline{f\left(\Lambda^{\infty}\right)}, \Lambda^{\infty}\right)$ is a cutand-project scheme. Therefore, both constructions are equivalent and we will use the latter as it is handier in our case. For further results on good models in groups see [13].

In [15] Yves Meyer proved a structure theorem for what later came to be known as mathematical quasi-crystals. Quasi-crystals correspond to uniform approximate lattices in locally compact abelian groups. Rephrased with our terminology, Meyer's theorem becomes :

Theorem 2 [15, Theorem 3.2] Let $\Lambda$ be a uniform approximate lattice in a locally compact abelian group $G$. Then $\Lambda$ is a Meyer subset.

Motivated by this theorem the authors of [2] asked whether similar results would hold for other classes of locally compact groups [2, Problem 1.]. We answer this question in the soluble Lie case. This generalises, using completely different methods, a previous article by the author that dealt with uniform approximate lattices in nilpotent Lie groups [12].

Theorem 3 Let $\Lambda \subset G$ be a uniform approximate lattice in a connected soluble Lie group. Then $\Lambda$ is a Meyer subset.

Let us now give a brief overview of the proof strategy for Theorems 1 and 3 .

Theorem 1 will be proved by induction on the derived length. We use induction to reduce the proof to the case where $\left\{\lambda_{1} \lambda_{2} \lambda_{1}^{-1} \lambda_{2}^{-1} \mid \lambda_{1}, \lambda_{2} \in \Lambda\right\}$ is relatively compact. Then we are able to show that $\Lambda$ is close to the centre of $\mathbb{G}(\mathbb{R})$. The crux of the proof relies on the following fact that is specific to algebraic group homomorphisms: if $\phi$ is an algebraic group homomorphism and $S$ is a set that has relatively compact image by $\phi$, then $S$ is contained in $\operatorname{ker}(\phi) K$ for some compact subset $K$. This is because $\phi$ has (Hausdorff) closed image and is a homeomorphism onto its image (as a consequence of e.g. [25, Propositions 3.16]). Applied to inner automorphisms, this yields a result reminiscent of a classical theorem of Schur, according to which a group with a finite set of commutators has a finite-index centre. 
In order to prove Theorem 3 , we first show that, although $\Lambda^{\infty}$ is a priori only a soluble group, $\Lambda$ is commensurable to a uniform approximate lattice $\Lambda^{\prime}$ that generates a polycyclic group. Using Auslander's embedding theorem on polycyclic groups we embed $\left(\Lambda^{\prime}\right)^{\infty}$ as a lattice in some soluble algebraic group. Then $\Lambda^{\prime}$ is a Meyer subset according to Theorem 1 .

\section{Technical results about commensurability}

In this section, we recall two technical results that will turn out to be particularly helpful. These are well-known results in the theory of finite approximate subgroups (see for instance [22]), but their proofs do not use the finiteness assumption.

Lemma 1 [22, Proposition 2.6.5] Let $\Lambda, \Xi \subset G$ be approximate subgroups. Then $\Lambda^{2} \cap \Xi^{2}$ is an approximate subgroup. Moreover, $\left(\Lambda^{k} \cap \Xi^{k}\right)_{k \geq 2}$ is a family of pairwise commensurable approximate subgroups.

We will often use Lemma 1 with $\Xi$ a subgroup. In this situation $\left(\Lambda^{k} \cap \Xi\right)_{k \geq 2}$ is a family of pairwise commensurable approximate subgroups. When considering commensurable approximate subgroups we can obtain further information. In particular, we can see that when $\Lambda$ and $\Xi$ are genuine subgroups, commensurability as defined here is equivalent to commensurability of subgroups.

Lemma 2 Let $\Lambda \subset G$ be an approximate subgroup and $X \subset G$ be a subset such that $\Lambda \subset F X$ for some finite set $F$. Then $\Lambda^{2} \cap X^{-1} X$ is an approximate subgroup commensurable to $\Lambda$.

Proof Let $F$ be a finite set such that $\Lambda \subset F X$ and assume that it is minimal for this property with respect to inclusion. For all $f \in F$ we can choose $x_{f} \in \Lambda \cap f X$. Then for any $x \in \Lambda \cap f X$ we have $x_{f}^{-1} x \in \Lambda^{2} \cap X^{-1} X$ so

$$
\Lambda \subset \bigcup x_{f}\left(\Lambda^{2} \cap X^{-1} X\right) \subset \Lambda^{3}
$$

Now $\left(\Lambda^{2} \cap X^{-1} X\right)$ is a symmetric subset containing $e$ and

$$
\Lambda^{2} \cap X^{-1} X \subset\left(\Lambda^{2} \cap X^{-1} X\right)^{2} \subset \Lambda^{4}
$$

But $\Lambda^{4}$ and $\Lambda^{2} \cap X^{-1} X$ are commensurable according to the first part of the proof. So $\left(\Lambda^{2} \cap X^{-1} X\right)^{2}$ is commensurable to $\Lambda^{2} \cap X^{-1} X$.

\section{Approximate subgroups in soluble linear groups}

In this section we prove Theorem 1. Let us first recall Schreiber's theorem about approximate subgroups in vector spaces. 
Theorem 4 [19, Proposition 2] Let $V$ be a real vector space and $\Lambda \subset V$ an approximate subgroup. There exists a vector subspace $W \subset V$ compactly commensurable to $\Lambda$.

We will extend Theorem 4 to soluble real algebraic groups. In the proof of the following proposition we rely on the theory of algebraic groups. See [25, §2 and 3] for a survey of the specific tools we will use and [20] for a general introduction to linear algebraic groups. Note that Proposition 1 below is in fact the main result of this paper. We will call upon Proposition 1 rather than Theorem 1 in the rest of the paper.

Proposition 1 Let $\Lambda \subset \mathbb{G}(\mathbb{R})$ be an approximate subgroup in the group of $\mathbb{R}$-points of a Zariski-connected soluble real algebraic group such that $\Lambda^{\infty}$ is Zariski-dense. Then there is a closed connected normal subgroup $H \triangleleft \mathbb{G}(\mathbb{R})$ such that $\Lambda$ is compactly commensurable to $H$.

The subgroup $H$ is the connected component of the identity of the group of $\mathbb{R}$-points of some normal algebraic subgroup $\mathbb{H}$ of $\mathbb{G}$. Moreover, there exists a unique minimal such $\mathbb{H}$ i.e. contained in every other normal algebraic subgroup $\mathbb{L}$ whose group of $\mathbb{R}$-points is compactly commensurable to $\Lambda$.

Proof We start with the uniqueness part. It will be an easy consequence of the following: take two normal algebraic subgroups $\mathbb{H}_{1}, \mathbb{H}_{2} \subset \mathbb{G}$ such that the projection of $\Lambda$ is relatively compact in $\mathbb{G}(\mathbb{R}) / \mathbb{H}_{i}(\mathbb{R})$ for $i=1,2$. Then the projection of $\Lambda$ through the diagonal group homomorphism $\Delta: \mathbb{G}(\mathbb{R}) \rightarrow\left(\mathbb{G} / \mathbb{H}_{1}\right)(\mathbb{R}) \times\left(\mathbb{G} / \mathbb{H}_{1}\right)(\mathbb{R})$ is relatively compact. But $\Delta$ is algebraic so there is a compact subset $K \subset \mathbb{G}(\mathbb{R})$ such that $\Lambda$ is contained in $K\left(\mathbb{H}_{1}(\mathbb{R}) \cap \mathbb{H}_{2}(\mathbb{R})\right.$ ) (as a consequence of $[25,3.16]$ ). In particular, if $\Lambda$ is compactly commensurable to $\mathbb{H}_{1}(\mathbb{R})$ and $\mathbb{H}_{2}(\mathbb{R})$, then $\Lambda$ is compactly commensurable to $\mathbb{H}_{1}(\mathbb{R}) \cap \mathbb{H}_{2}(\mathbb{R})$. Now, by the descending chain condition for Zariski-closed subsets of $\mathbb{G}(\mathbb{R})$ there is a Zariski-closed normal subgroup compactly commensurable to $\Lambda$ that does not contain any Zariski-closed normal proper subgroup compactly commensurable to $\Lambda$. So by the discussion it must be contained in every other Zariski-closed normal subgroup compactly commensurable to $\Lambda$.

Let us now move on to proving existence. As $\Lambda^{\infty}$ is Zariski-dense, we know that $\Lambda^{\infty} \cap[\mathbb{G}(\mathbb{R}), \mathbb{G}(\mathbb{R})]$ is Zariski-dense in $[\mathbb{G}(\mathbb{R}), \mathbb{G}(\mathbb{R})]$. Moreover, $[\mathbb{G}(\mathbb{R}), \mathbb{G}(\mathbb{R})]$ is a connected simply connected nilpotent Lie group so $\overline{\Lambda^{\infty} \cap[\mathbb{G}(\mathbb{R}), \mathbb{G}(\mathbb{R})]}$ is cocompact by [17, Theorem 2.1]. As a consequence, there is $k \in \mathbb{N}$ greater than 2 such that $\Lambda^{\prime}:=\Lambda^{k} \cap[\mathbb{G}(\mathbb{R}), \mathbb{G}(\mathbb{R})]$ with $\Lambda^{\prime \infty}$ Zariski-dense in $[\mathbb{G}(\mathbb{R}), \mathbb{G}(\mathbb{R})]$. By Lemma 1 we know that $\Lambda^{\prime}$ is an approximate subgroup. According now to the induction hypothesis there is a closed connected subgroup $H_{1} \triangleleft[\mathbb{G}(\mathbb{R}), \mathbb{G}(\mathbb{R})]$ compactly commensurable to $\Lambda^{\prime}$. In addition, for all $\lambda \in \Lambda$, we have $\lambda\left(\Lambda^{\prime}\right) \lambda^{-1} \subset \Lambda^{k+2} \cap[\mathbb{G}(\mathbb{R}), \mathbb{G}(\mathbb{R})]$. But, according to Lemma 1 approximate subgroups $\Lambda^{k+2} \cap[\mathbb{G}(\mathbb{R}), \mathbb{G}(\mathbb{R})]$ and $\Lambda^{\prime}$ are commensurable. Therefore, $H_{1}$ and $\lambda H_{1} \lambda^{-1}$ are compactly commensurable. We thus have $H_{1}=\lambda H_{1} \lambda^{-1}$ (see Lemma 3 below).

Now $H_{1}$ is connected, so its normaliser $N\left(H_{1}\right)$ is equal to the stabiliser of its Lie algebra. But then $N\left(H_{1}\right)$ is Zariski-closed and contains $\Lambda$ which is Zariski dense. So $N\left(H_{1}\right)=\mathbb{G}(\mathbb{R})$ and $H_{1}$ is normal. Since $H_{1}$ is connected in a unipotent subgroup, $H_{1}$ is the group of $\mathbb{R}$-points of an algebraic subgroup $\mathbb{H}_{1}$ normal in $\mathbb{G}$ (e.g. [25, Lemma 3.20]). Therefore, the natural map $\mathbb{G}(\mathbb{R}) / \mathbb{H}_{1}(\mathbb{R}) \rightarrow\left(\mathbb{G} / \mathbb{H}_{1}\right)(\mathbb{R})$ is an embedding and 
its image contains the connected component of the identity in $\left(\mathbb{G} / \mathbb{H}_{1}\right)(\mathbb{R})$. Moreover, the Zariski-closure of the image of $\Lambda^{\infty}$ contains the image of $\mathbb{G}(\mathbb{R})$.

Set $p: \mathbb{G}(\mathbb{R}) \rightarrow\left(\mathbb{G} / \mathbb{H}_{1}\right)(\mathbb{R})$ the canonical projection, $\tilde{\Lambda}:=p\left(\Lambda^{2}\right)$ and $\tilde{G}$ the Zariski-closure of $\tilde{\Lambda}$.

Claim The approximate subgroup $\tilde{\Lambda}$ is compactly commensurable to a Zariski-closed subgroup of the centre $Z(\tilde{G})$ of $\tilde{G}$.

Let us first show how Proposition 1 follows from this claim. There is a Zariskiclosed subgroup $V \subset Z(\tilde{G})$ such that $\tilde{\Lambda}$ is compactly commensurable to $V$. So we can find a compact subset $K_{1} \subset \mathbb{G}(\mathbb{R})$ such that

$$
\tilde{\Lambda} \subset p\left(K_{1}\right) V \quad \text { and } \quad V \subset p\left(K_{1}\right) \tilde{\Lambda}
$$

According to the first inclusion we have $\Lambda \subset K_{1} p^{-1}(V)$. On the other hand, there is $K_{2} \subset \mathbb{G}(\mathbb{R})$ compact such that $H_{1} \subset K_{2} \Lambda$, where $H_{1}$ is the subgroup defined above. Finally,

$$
p^{-1}(V) \subset K_{1} \Lambda H_{1}=K_{1} H_{1} \Lambda \subset K_{1} K_{2} \Lambda^{2}
$$

So the subgroup $p^{-1}(V)$ is compactly commensurable to $\Lambda$. But $p^{-1}(V)$ is equal to the $\mathbb{R}$-points of an algebraic subgroup $\mathbb{H} \subset \mathbb{G}$ since both $V$ and $H_{1}$ are Zariskiclosed subgroups. The subgroup $V$ being central implies furthermore that $\mathbb{H}$ is normal. Finally, we know that $\mathbb{H}(\mathbb{R})$ has finitely many connected components [23], so denoting by $H$ the connected component of the identity in $\mathbb{H}(\mathbb{R})$ we indeed find a subgroup satisfying the conclusions of Proposition 1.

Now let us move to the proof of the claim. We will use the fact that the set of commutators of elements of $\tilde{\Lambda}$ is relatively compact to show that $\tilde{\Lambda}$ is contained in a 'neighbourhood' of the centre. The group $\tilde{\Lambda}^{\infty} \cap[\tilde{G}, \tilde{G}]$ is co-compact in $[\tilde{G}, \tilde{G}]$. So there is $l \in \mathbb{N}$ such that

$$
\operatorname{span}_{\mathbb{R}}\left(\log \left(\tilde{\Lambda}^{l} \cap[\tilde{G}, \tilde{G}]\right)\right)=\mathfrak{L} \mathfrak{i} \mathfrak{e}([\tilde{G}, \tilde{G}])
$$

where $\log$ is the logarithm map from $[\tilde{G}, \tilde{G}]$ to its Lie algebra. In addition,

$$
\bigcup_{\lambda \in \tilde{\Lambda}} \lambda\left(\tilde{\Lambda}^{l} \cap[\tilde{G}, \tilde{G}]\right) \lambda^{-1} \subset \tilde{\Lambda}^{l+2} \cap[\tilde{G}, \tilde{G}] .
$$

But by definition of $H_{1}$ we know that $\tilde{\Lambda} \cap[\tilde{G}, \tilde{G}]$ is relatively compact. And thanks to Lemma 1 and since $\tilde{\Lambda}=p(\Lambda)^{2}$ the subset $\tilde{\Lambda}^{r} \cap[\tilde{G}, \tilde{G}]$ is an approximate subgroup commensurable to $\tilde{\Lambda} \cap[\tilde{G}, \tilde{G}]$ for all $r \in \mathbb{N}$. The right-hand side of the above inclusion is therefore a relatively compact subset. Hence, the family of linear operators $\left(\operatorname{Ad}(\lambda)_{\mid \mathfrak{L} \mathfrak{i}([\tilde{G}, \tilde{G}])}\right)_{\lambda \in \Lambda}$ is uniformly bounded. Since

$$
\begin{aligned}
\rho:(\mathbb{G} / \mathbb{H})(\mathbb{R}) & \rightarrow \operatorname{GL}(\mathfrak{L} \mathfrak{i e}([\tilde{G}, \tilde{G}])) \\
g & \mapsto \operatorname{Ad}(g)_{\mid \mathfrak{L i e}([\tilde{G}, \tilde{G}])}
\end{aligned}
$$


is an algebraic group homomorphism, there is a compact set $K \subset \tilde{G}$ such that

$$
\tilde{\Lambda} \subset \operatorname{ker}(\rho) K .
$$

In the following we will denote $\operatorname{ker}(\rho)$ by $Z$. Note that since $[\tilde{G}, \tilde{G}]$ is connected, $Z$ is in fact the centraliser of $[\tilde{G}, \tilde{G}]$.

Now for any $g \in \tilde{G}$ define the map

$$
\begin{aligned}
\theta_{g}: Z & \rightarrow \tilde{G} \\
h & \mapsto[g, h],
\end{aligned}
$$

where $[g, h]$ denotes $g h g^{-1} h^{-1}$. For $h_{1}, h_{2} \in Z$ we have

$$
\begin{aligned}
\theta_{g}\left(h_{1}\right) \theta_{g}\left(h_{2}\right) & =g h_{1} g^{-1} h_{1}^{-1} g h_{2} g^{-1} h_{2}^{-1} \\
& =g h_{1} g^{-1} g h_{2} g^{-1} h_{2}^{-1} h_{1}^{-1} \\
& =g\left(h_{1} h_{2}\right) g^{-1}\left(h_{1} h_{2}\right)^{-1} \\
& =\theta_{g}\left(h_{1} h_{2}\right) .
\end{aligned}
$$

So $\theta_{g}$ is an algebraic group homomorphism.

For all $\lambda \in \tilde{\Lambda}$ let $f(\lambda)$ denote an element of $Z$ such that $\delta(\lambda):=\lambda f(\lambda)^{-1} \in K_{3}$. Now, for $\gamma \in \tilde{\Lambda}$ and $\lambda \in \tilde{\Lambda}$ we have

$$
\begin{aligned}
\theta_{\gamma}(f(\lambda)) & =\gamma f(\lambda) \gamma^{-1} f(\lambda)^{-1} \\
& =\gamma \delta(\lambda)^{-1} \lambda \gamma^{-1}\left(\delta(\lambda)^{-1} \lambda\right)^{-1} \\
& =\left(\gamma \delta(\lambda)^{-1} \gamma^{-1}\right) \gamma \lambda \gamma^{-1} \lambda^{-1}(\delta(\lambda))
\end{aligned}
$$

In particular

$$
\theta_{\gamma}(f(\tilde{\Lambda})) \subset \gamma K_{3}^{-1} \gamma^{-1}\left(\tilde{\Lambda}^{4} \cap[\tilde{G}, \tilde{G}]\right) K_{3}
$$

So $\theta_{\gamma}(f(\tilde{\Lambda}))$ is a relatively compact set.

Set now

$$
\begin{aligned}
\theta_{\Upsilon}: Z & \rightarrow(Z)^{n} \\
g & \mapsto\left(\theta_{\gamma_{1}}(g), \ldots, \theta_{\gamma_{n}}(g)\right)
\end{aligned}
$$

where $\Upsilon$ is any finite family $\left\{\gamma_{1}, \ldots, \gamma_{n}\right\}$ of elements of $\tilde{\Lambda}$. We readily see that

$$
\operatorname{ker}\left(\theta_{\Upsilon}\right)=\bigcap_{1 \leq i \leq n} Z_{\tilde{G}}\left(\gamma_{i}\right) \cap Z .
$$

We know that $Z$ is an algebraic subgroup and $\theta_{\Upsilon}$ is an algebraic group morphism. Moreover, since $\theta_{\Upsilon}(f(\tilde{\Lambda}))$ is relatively compact as a subset of $\tilde{G}^{n}$ and $\theta_{\Upsilon}(Z)$ is 
closed, it is relatively compact as a subset of $\theta_{\Upsilon}(Z)$. Thus, there is a compact set $K_{4}$ such that $f(\tilde{\Lambda}) \subset K_{4} \operatorname{ker}\left(\theta_{\Upsilon}\right)$. Setting $K_{\Upsilon}:=K_{3} K_{4}$, we get $\tilde{\Lambda} \subset K_{\Upsilon} \operatorname{ker}\left(\theta_{\Upsilon}\right)$.

Since $\tilde{\Lambda}^{\infty}$ is Zariski-dense, the centraliser of elements are Zariski-closed and Zariski-closed subsets satisfy the descending chain condition, we can find $\Upsilon \subset \tilde{\Lambda}^{\infty}$ finite such that the centraliser of $\Upsilon$ is equal to the centre $Z(\tilde{G})$ of $\tilde{G}$. Hence,

$$
\tilde{\Lambda} \subset K_{\Upsilon} \operatorname{ker}\left(\theta_{\Upsilon}\right)=K_{\Upsilon} Z(\tilde{G})
$$

But $Z(\tilde{G})$ is a Zariski-closed subgroup, so it has a finite number of connected components [23]. Besides, the connected component of the identity is isomorphic to $\mathbb{R}^{k} \times \mathbb{T}^{l}$ for some $k, l \in \mathbb{N}$. There is therefore a central subgroup $W \subset \tilde{G}$ and a compact subset $K_{5} \subset \tilde{G}$ such that $\tilde{\Lambda} \subset K_{5} W$ and $W \simeq \mathbb{R}^{k}$

Finally, choose a function $g: \tilde{\Lambda} \rightarrow W$ such that for all $\lambda \in \tilde{\Lambda}, g\left(\lambda^{-1}\right)=g(\lambda)^{-1}$ and $b(\lambda):=g(\lambda) \lambda^{-1} \in K_{5}$. There is a finite subset $F \subset G$ such that for all $\lambda_{1}, \lambda_{2} \in \tilde{\Lambda}$ there is $\lambda \in \tilde{\Lambda}$ satisfying $\lambda \lambda_{1}^{-1} \lambda_{2}^{-1} \in F$. Relying on the centrality of $W$ we compute

$$
\begin{aligned}
g(\lambda) g\left(\lambda_{1}\right)^{-1} g\left(\lambda_{2}\right)^{-1} & =b(\lambda) \lambda \lambda_{1}^{-1} b\left(\lambda_{1}\right)^{-1} g\left(\lambda_{2}\right)^{-1} \\
& =\lambda \lambda_{1}^{-1} b\left(\lambda_{1}\right)^{-1} g\left(\lambda_{2}\right)^{-1} b(\lambda) \\
& =\lambda \lambda_{1}^{-1} g\left(\lambda_{2}\right)^{-1} b\left(\lambda_{1}\right)^{-1} b(\lambda) \\
& =\lambda \lambda_{1}^{-1} \lambda_{2}^{-1} b\left(\lambda_{2}\right)^{-1} b\left(\lambda_{1}\right)^{-1} b(\lambda) .
\end{aligned}
$$

And we find

$$
g(\lambda) g\left(\lambda_{1}\right)^{-1} g\left(\lambda_{2}\right)^{-1} \in F K_{5}^{-2} K_{5}
$$

We have thus proved that the subset $g(\tilde{\Lambda})$ is such that $g(\tilde{\Lambda})+g(\tilde{\Lambda})$ is compactly commensurable to $g(\tilde{\Lambda})$. We then have that $g(\tilde{\Lambda})$ is compactly commensurable to an approximate subgroup (see Lemma 4 below). By Schreiber's theorem (Theorem 4) there is therefore a closed connected subgroup $V_{1}$ compactly commensurable to $g(\tilde{\Lambda})$ - hence to $\tilde{\Lambda}$. But $V_{1} \subset Z(\tilde{G})$ is compactly commensurable to its Zariski-closure $V_{2}$ (see [24, Cororollary 4.1]). So $V_{2}$ is compactly commensurable to $\tilde{\Lambda}$.

We prove now the two technical lemmas used in the proof of Proposition 1.

Lemma 3 Let $H_{1}, H_{2}$ be two connected subgroups of a simply connected nilpotent group $N$. Then $\mathrm{H}_{1}$ and $\mathrm{H}_{2}$ are compactly commensurable if and only if they are equal.

Proof Let $K \subset N$ be a compact subset such that $H_{2} \subset H_{1} K$. We proceed by induction on the length of the upper central series. If $N \simeq \mathbb{R}^{n}$ for some $n \in \mathbb{N}$, the result is obvious. Otherwise let $Z$ be the centre of $N$, by induction hypothesis the projections of $H_{1}$ and $H_{2}$ to $N / Z$ are equal. So given $g \in H_{2}$, there is $z \in Z$ such that $g z \in H_{1}$. Moreover for all $n \in \mathbb{N}$ there are $h_{n} \in H_{1}$ and $k_{n} \in K$ such that $g^{n}=h_{n} k_{n}$. Hence,

$$
\forall n \in \mathbb{N}, z^{n} k_{n}=z^{n} h_{n}^{-1} g^{n}=h_{n}^{-1}(g z)^{n} \in H_{1}
$$


So,

$$
\forall n \in \mathbb{N}, \log (z)+\frac{\log \left(k_{n}\right)}{n} \in \log \left(H_{1}\right)
$$

where $\log$ is the logarithm map from $N$ to its Lie alebra. But $\log \left(H_{1}\right)$ is closed (since it is a vector subspace of the Lie algebra) so we find $z \in H_{1}$ and $g \in H_{1}$.

Lemma 4 Let $A$ be a locally compact abelian group and $\Lambda \subset V$ a symmetric subset such that $\Lambda+\Lambda$ is compactly commensurable to $\Lambda$. Then $\Lambda$ is compactly commensurable to an approximate subgroup.

Proof Let $U \subset V$ be a symmetric compact neighbourhood of 0 , then $\Lambda+U$ is a symmetric set compactly commensurable to $\Lambda$. Moreover, let $K \subset V$ be a compact subset such that $\Lambda+\Lambda \subset \Lambda+K$ and $F \subset V$ be a finite subset such that $K+U+U \subset$ $U+F$. Then we have,

$$
(\Lambda+U)+(\Lambda+U) \subset \Lambda+K+U+U \subset(\Lambda+U)+F .
$$

Proof of Theorem 1 Let $G$ be the Zariski-closure of $\Lambda^{\infty}$. Then $G$ is the group of $\mathbb{R}$ points of a soluble algebraic group. Let $\tilde{G}$ denote the group of $\mathbb{R}$-points of its Zariskiconnected component of the identity. The subgroup $\Lambda^{\infty} \cap \tilde{G}$ is Zariski-dense in $\tilde{G}$ so we can find a finite subset $X \subset \Lambda^{\infty} \cap \tilde{G}$ such that $X \tilde{G}=G$. There is therefore an integer $n \geq 1$ such that $X \subset \Lambda^{n} \cap \tilde{G}$; a classical argument-found e.g. in [11] about generation in finite index subgroups now shows that $\tilde{G} \cap \Lambda^{\infty}$ is generated by $\Lambda^{2 n+1} \cap \tilde{G}$. By Lemma 1 , the set $\Lambda^{2 n+1} \cap \tilde{G}$ is an approximate subgroup. Applying Proposition 1 to $\Lambda^{2 n+1} \cap \tilde{G}$ we get a minimal Zariski-closed normal subgroup $H$ of $\tilde{G}$ that is compactly commensurable to $\Lambda^{2 n+1} \cap \tilde{G}$. But $\Lambda^{2 n+1} \cap \tilde{G}$ is commensurable to $\Lambda^{2} \cap \tilde{G}$ according to Lemma 1 and $\Lambda^{2} \cap \tilde{G}$ is commensurable to $\Lambda$ by Lemma 2 . The subgroup $H$ is thus compactly commensurable to $\Lambda$. Now for any $\lambda \in \Lambda$ notice that $\lambda\left(\Lambda^{2 n+1} \cap \tilde{G}\right) \lambda^{-1}=\lambda\left(\Lambda^{2 n+1}\right) \lambda^{-1} \cap \tilde{G}$ is commensurable to $\Lambda^{2 n+1} \cap \tilde{G}$ according to Lemma 1. So $\lambda H \lambda^{-1}$ is a normal Zariski-closed subgroup of $\tilde{G}$ and is compactly commensurable to $\lambda\left(\Lambda^{2 n+1} \cap \tilde{G}\right) \lambda^{-1}$-hence to $\Lambda^{2 n+1} \cap \tilde{G}$. By minimality of $H$ we find $H \subset \lambda H \lambda^{-1}$. The inclusion being true for all elements in $\Lambda$, we have that $H$ is normalised by $\Lambda^{\infty}$ and by its Zariski-closure.

\section{Consequences of Theorem 1}

In [8] Fried and Goldman proved that every soluble subgroup $\Gamma$ of $\mathrm{GL}_{n}(\mathbb{R})$ admits a syndetic hull. More precisely:

Proposition 2 [8, Theorem 1.6] Let $\mathbb{G}$ be a soluble real algebraic group and $\Gamma \subset \mathbb{G}(\mathbb{R})$ a subgroup. Then there is $H<G$ such that $H$ is a closed connected subgroup (in 
the Euclidean topology), $\Gamma \cap H$ has finite index in $\Gamma$ and $\Gamma$ and $H$ are compactly commensurable.

We show how this theorem is in fact a consequence of Theorem 1.

Proof Without loss of generality we can assume that $\Gamma$ is Zariski-dense. Let $\mathbb{G}^{0}$ be the Zariski-connected component of the identity of $\mathbb{G}$. The subgroup $\Gamma \cap \mathbb{G}^{0}(\mathbb{R})$ has finite index in $\Gamma$ and is Zariski-dense in $\mathbb{G}^{0}(\mathbb{R})$. Then applying Proposition 1 to $\Gamma \cap \mathbb{G}^{0}(\mathbb{R})$ we get a closed connected normal subgroup $H \subset \mathbb{G}^{0}(\mathbb{R})$ such that $\Gamma$ is compactly commensurable to $H$. So the image of $\Gamma \cap \mathbb{G}^{0}(\mathbb{R})$ in $\mathbb{G}^{0}(\mathbb{R}) / H$ via $p: \mathbb{G}^{0}(\mathbb{R}) \rightarrow \mathbb{G}^{0}(\mathbb{R}) / H$ is contained in a compact subgroup $K$. Let $K_{0}$ be the connected component of the identity of $K$ in the Euclidean topology, then set $\tilde{\Gamma}=\Gamma \cap p^{-1}\left(K_{0}\right)$. The subgroup $\tilde{\Gamma}$ has finite index in $\Gamma \cap \mathbb{G}^{0}(\mathbb{R})$, hence in $\Gamma$, and is co-compact in the closed connected subgroup $p^{-1}\left(K_{0}\right)$.

We also get a generalisation of the well-known fact that closed soluble subgroups of $\mathrm{GL}_{n}(\mathbb{R})$ are compactly generated. Note that in Proposition 3 below we do not assume the approximate subgroup $\Lambda$ considered to be closed. Specialised to (not necessarily closed) soluble subgroups of $\mathrm{GL}_{n}(\mathbb{R})$ our conclusion is that any soluble subgroup is generated by a subset that is relatively compact in $\mathrm{GL}_{n}(\mathbb{R})$.

Proposition 3 Let $G$ be the $\mathbb{R}$-points of a soluble real algebraic group and $\Lambda \subset G$ an approximate subgroup. Then there is a compact subset $K$ such that $\Lambda^{2} \cap K$ generates $\Lambda^{\infty}$.

Proof Let $H$ be the Zariski-closure of $\Lambda^{\infty}$. Proceeding as in the proof of Theorem 1 we find an integer $k \geq 2$ such that $\Lambda^{\prime}:=\Lambda^{k} \cap H^{0}$ generates a Zariski-dense subgroup of the Zariski-connected component of the identity $H^{0}$ of $H$. Note moreover that $\Lambda^{\prime}$ is an approximate subgroup commensurable to $\Lambda$ (Lemma 2). Applying Proposition 1 to $\Lambda^{\prime}$ we find a connected subgroup $H \leq G$ such that $G$ and $\Lambda^{\prime}$ are compactly commensurable. Since $\Lambda$ and $\Lambda^{\prime}$ are commensurable, there is a compact symmetric subset $K \subset G$ such that $\Lambda \subset K H$ and $H \subset K \Lambda$. Choose also $V$ a compact neighbourhood of the identity in $H$. As $H$ is connected for the Euclidean topology, $V$ generates $H$. Now, for any $\lambda \in \Lambda$ choose $h \in H$ such that $\lambda h^{-1} \in K$. Since $V$ generates $H$, we can find a sequence $\left(h_{i}\right)_{0 \leq i \leq r}$ of elements of $H$ such that $h_{0}=e, h_{r}=h$ and $h_{i+1} h_{i}^{-1} \in V$. In addition, we can find a sequence $\left(\lambda_{i}\right)_{0<i<n}$ of elements of $\Lambda$ such that $\lambda_{0}=e, \lambda_{r}=\lambda$ and for all $0 \leq i \leq r, \lambda_{i} h_{i}^{-1} \in K^{-1}=K$. Thus, $\lambda_{i+1} \lambda_{i}^{-1} \in K V^{-1} K^{-1}$. Finally, $\Lambda^{\infty}$ is generated by $\Lambda^{2} \cap K V^{-1} K^{-1}$.

Corollary 1 If $\Lambda$ is discrete, then $\Lambda^{\infty}$ is finitely generated.

Finally, we give a partial generalisation of a theorem from [2, Theorem 4.25], where it was proved that weak approximate lattices in nilpotent locally compact groups are uniform. Our result is concerned with the more restrictive class of approximate lattices in soluble real algebraic groups. Approximate lattices are defined by measure-theoretic conditions on an associated dynamical system called the invariant hull. We refer the reader to [2, Section 4] for precise definitions. 
Theorem 5 Let $\Lambda \subset G$ be a strong approximate lattice in the group of $\mathbb{R}$-points of a connected soluble real algebraic group. Then $\Lambda$ is relatively dense.

Proof Indeed, according to [2, Remark 4.14.(1) \& Theorem 4.18] any approximate lattice is bi-syndetic in an amenable group i.e. there is $K_{1} \subset G$ compact such that $G=K_{1} \Lambda K_{1}$. Moreover, $\Lambda$ is Zariski-dense according to [3]. Now let $H$ and $K_{2}$ be given by Proposition 1 so that

$$
\Lambda \subset K_{2} H \text { and } H \subset K_{2} \Lambda \text {. }
$$

Since $H$ is normal we have that $G=K_{1} K_{2} H K_{1}=K_{1} K_{2} K_{1} H$. Then $G=$ $K_{1} K_{2} K_{1} K_{2} \Lambda$.

\section{Uniform approximate lattices in abelian groups}

We will investigate morphisms commensurating approximate subgroups in $\mathbb{R}^{n}$. This will turn out to be useful in the proof of Theorem 3. Our goal is to understand morphisms that commensurate a uniform approximate lattice. Let us start with a result concerning lattices.

Proposition 4 Let $\Lambda \subset \mathrm{GL}_{n}(\mathbb{R})$ be an approximate subgroup and suppose there are $\Gamma_{1} \subset \Gamma_{2}$ lattices in $\mathbb{R}^{n}$ such that $\lambda\left(\Gamma_{1}\right) \subset \Gamma_{2}$ for all $\lambda \in \Lambda$. Then there is an approximate subgroup $\Xi \subset \Lambda^{4}$ commensurable to $\Lambda$ such that $\Xi \subset \operatorname{Aut}\left(\Gamma_{1}\right)$.

Proof We can assume that $\Gamma_{1}=\mathbb{Z}^{n}$. Let $m$ be the order of $\Gamma_{2} / \Gamma_{1}$ and $p_{1}, \ldots, p_{r}$ the prime factors of $m$. Then any matrix in $\Lambda$ has entries lying in $\frac{1}{m} \mathbb{Z}$.

Set

$$
\begin{aligned}
\phi: \mathrm{GL}_{n}(\mathbb{R}) & \rightarrow \mathbb{R}_{+}^{*} \\
M & \mapsto|\operatorname{det}(M)|
\end{aligned}
$$

then $\phi$ is a group homomorphism and $\phi(\Lambda) \subset \frac{1}{m^{n}} \mathbb{Z}$ is a discrete approximate subgroup bounded away from 0 so $\phi(\Lambda)$ is finite. As a consequence,

$$
\tilde{\Lambda}=\phi^{-1}(\{1\}) \cap \Lambda^{2}
$$

is an approximate subgroup commensurable to $\Lambda$ by Lemma 2 .

Consider the diagonal embedding

$$
\iota: \mathrm{SL}_{n}\left(\mathbb{Z}\left[\frac{1}{m}\right]\right) \hookrightarrow \prod_{i=1}^{r} \mathrm{SL}_{n}\left(\mathbb{Q}_{p_{i}}\right)
$$


Now $\iota(\tilde{\Lambda})$ is relatively compact and $\prod_{i=1}^{r} \operatorname{SL}_{n}\left(\mathbb{Z}_{p_{i}}\right)$ is open. Therefore, there are $\lambda_{1}, \ldots, \lambda_{s} \in \tilde{\Lambda}$ such that

$$
\iota(\tilde{\Lambda}) \subset \bigcup_{i=1}^{s} \iota\left(\lambda_{s}\right)\left(\prod_{i=1}^{r} \operatorname{SL}_{n}\left(\mathbb{Z}_{p_{i}}\right)\right)
$$

So

$$
\tilde{\Lambda}^{2} \cap \iota^{-1}\left(\prod_{i=1}^{r} \mathrm{SL}_{n}\left(\mathbb{Z}_{p_{i}}\right)\right)
$$

is an approximate subgroup commensurable to $\tilde{\Lambda}$ by Lemma 2 . Since $\iota^{-1}\left(\prod_{i=1}^{r} \mathrm{SL}_{n}\right.$ $\left.\left(\mathbb{Z}_{p_{i}}\right)\right)=\operatorname{SL}_{n}(\mathbb{Z})$, we can set $\Xi:=\tilde{\Lambda}^{2} \cap \mathrm{SL}_{n}(\mathbb{Z})$.

Now, we can deduce

Proposition 5 Let $\Lambda \subset \mathrm{GL}_{n}(\mathbb{R})$ be an approximate subgroup and suppose there are $\Lambda_{1} \subset \Lambda_{2}$ approximate lattices in $\mathbb{R}^{n}$ such that $\lambda\left(\Lambda_{1}\right) \subset \Lambda_{2}$ for all $\lambda \in \Lambda$. Then there are $\Xi \subset \Lambda^{4}$ commensurable to $\Lambda$ and an injective group homomorphism $\Xi^{\infty} \rightarrow$ $\mathrm{SL}_{m}(\mathbb{Z})$ for some $m \geq n$.

This result is not needed in the sequel, however it gives a good insight into the remaining part of the proof of Theorem 3. Indeed, a similar argument will be used to prove Proposition 7.

Proof According to Corollary 1 any approximate subgroup commensurable to $\Lambda_{1}$ generates a subgroup with finite rank. Take $\Lambda_{0}$ commensurable to $\Lambda_{1}$ generating a group of minimal rank. By Lemma 2 the approximate subgroup $\Lambda_{0}^{2} \cap \Lambda_{1}^{2}$ is commensurable to $\Lambda_{1}$. But $\operatorname{rank}\left(\left(\Lambda_{0}^{2} \cap \Lambda_{1}^{2}\right)^{\infty}\right) \leq \operatorname{rank}\left(\Lambda_{0}^{\infty}\right)$, so there is equality of ranks. We will therefore assume that $\Lambda_{0} \subset \Lambda_{1}^{2}$. We also know that for all $\lambda \in \Lambda$ the approximate group $\lambda\left(\Lambda_{0}\right)$ is commensurable to $\lambda\left(\Lambda_{1}\right)$ which in turn is commensurable to $\Lambda_{2}$. So $\Lambda_{0}$ and $\lambda\left(\Lambda_{0}\right)$ are commensurable. Hence, $\Lambda_{0}$ is commensurable to $\Lambda_{0}^{2} \cap \lambda\left(\Lambda_{0}^{2}\right)$ (Lemma 2$)$. Also, minimality of $\operatorname{rank}\left(\Lambda_{0}^{\infty}\right)$ yields

$$
\operatorname{rank}\left(\left(\Lambda_{0}^{2} \cap \lambda\left(\Lambda_{0}^{2}\right)\right)^{\infty}\right)=\operatorname{rank}\left(\Lambda_{0}^{\infty}\right)=\operatorname{rank}\left(\lambda\left(\Lambda_{0}^{\infty}\right)\right)
$$

So $\Lambda_{0}^{2} \cap \lambda\left(\Lambda_{0}^{2}\right)$ generates a finite index subgroup of $\Lambda_{0}^{\infty}$.

Therefore, $\lambda$ is an isomorphism of the $\mathbb{Q}$-span $\operatorname{span}_{\mathbb{Q}}\left(\Lambda_{0}^{\infty}\right)$ of $\Lambda_{0}^{\infty}$. Choosing a basis of $\operatorname{span}_{\mathbb{Q}}\left(\Lambda_{0}^{\infty}\right)$ adapted to the subgroup $\Lambda_{0}^{\infty}$ we thus have a group homomorphism $\phi: \Lambda^{\infty} \rightarrow \mathrm{GL}_{m}(\mathbb{Q})$ where $m=\operatorname{rank}\left(\Lambda_{0}^{\infty}\right)$. Since $\operatorname{span}_{\mathbb{R}}\left(\Lambda_{0}^{\infty}\right)=\mathbb{R}^{n}$ this group homomorphism is injective. Now, for all $\lambda \in \Lambda$ we have $\lambda\left(\Lambda_{0}^{\infty}\right) \subset \Lambda_{2}^{\infty} \cap \operatorname{span}_{\mathbb{Q}}\left(\Lambda_{0}^{\infty}\right)$. But

$$
\operatorname{rank}\left(\Lambda_{0}^{\infty}\right) \leq \operatorname{rank}\left(\Lambda_{2}^{\infty} \cap \operatorname{span}_{\mathbb{Q}}\left(\Lambda_{0}^{\infty}\right)\right) \leq \operatorname{dim}_{\mathbb{Q}}\left(\operatorname{span}_{\mathbb{Q}}\left(\Lambda_{0}^{\infty}\right)\right)=\operatorname{rank}\left(\Lambda_{0}^{\infty}\right)
$$


So $\Lambda_{0}^{\infty}$ has finite index in $\Lambda_{2}^{\infty} \cap \operatorname{span}_{\mathbb{Q}}\left(\Lambda_{0}^{\infty}\right)$. So Proposition 4 applied to $\Lambda_{0}^{\infty}, \Lambda_{2}^{\infty} \cap$ $\operatorname{span}_{\mathbb{Q}}\left(\Lambda_{0}^{\infty}\right)$ and $\Lambda$ gives an approximate subgroup $\Xi$ commensurable to $\Lambda$ that consists of automorphisms of $\Lambda_{0}^{\infty}$. Rewording the last statement using $\phi$ we find

$$
\phi(\Xi) \subset \mathrm{SL}_{m}(\mathbb{Z})
$$

Remark 1 From the proof of Proposition 5, we have that for any discrete approximate lattice $\Lambda \subset \mathbb{R}^{n}$ the subgroup $\left\{g \in \mathrm{GL}_{n}(\mathbb{R}) \mid g(\Lambda)\right.$ is commensurable to $\left.\Lambda\right\}$ is isomorphic to a subgroup of $\mathrm{GL}_{m}(\mathbb{Q})$ where $m$ is the minimal rank of an approximate subgroup commensurable to $\Xi$.

\section{Meyer's Theorem for soluble Lie groups}

We will now turn to the proof of Theorem 3. Our first step towards this goal is to prove a version under an additional assumption.

Proposition 6 Let $\Lambda \subset G$ be a uniform approximate lattice in a connected soluble Lie group. If $\Lambda^{\infty}$ is polycyclic then $\Lambda$ is a Meyer subset.

Proof According to a theorem of Auslander (see [1] or the proof of [17, Theorem 4.28]), $\Lambda^{\infty}$ admits an embedding as a Zariski-dense lattice in $R$ the group of $\mathbb{R}$-points of a soluble algebraic group. In the following we will consider $\Lambda^{\infty}$ as a subgroup of $R$. Moreover, we can assume without loss of generality that $R$ is Zariski-connected. Indeed, there is a finite index subgroup $\Gamma$ of $\Lambda^{\infty}$ such that the Zariski closure of $\Gamma$ is Zariski-connected. Furthermore, proceeding as in the proof of Theorem 1 we can find $n \in \mathbb{N}$ such that $\Lambda^{n} \cap \Gamma$ is an approximate subgroup commensurable to $\Lambda$ (Lemma 2) and generates $\Gamma$.

Now according to Proposition 1 there is a closed connected normal subgroup $N \triangleleft R$ such that $\Lambda$ is compactly commensurable to $N$. Let $p: R \rightarrow R / N$ denote the natural projection. We know that $p(\Lambda)$ is relatively compact, so we can choose a symmetric compact neighbourhood $W_{0}$ of $p(\Lambda)$. Now $p^{-1}\left(W_{0}\right)$ is an approximate subgroup compactly commensurable to $N$. But $\Lambda \subset \Lambda^{\infty} \cap p^{-1}\left(W_{0}\right)$ so both subsets are compactly commensurable to the subgroup $N$. So $\Lambda$ and $\Lambda^{\infty} \cap p^{-1}\left(W_{0}\right)$ are compactly commensurable i.e. there is a compact subset $K \subset R$ such that

$$
\Lambda^{\infty} \cap p^{-1}\left(W_{0}\right) \subset K \Lambda
$$

Since $\Lambda$ and $\Lambda^{\infty} \cap p^{-1}\left(W_{0}\right)$ are moreover contained in $\Lambda^{\infty}$ we have

$$
\Lambda^{\infty} \cap p^{-1}\left(W_{0}\right) \subset\left(K \cap \Lambda^{\infty}\right) \Lambda .
$$

But $\Lambda^{\infty}$ is a discrete subgroup of $R$ so $K \cap \Lambda^{\infty}$ is finite - meaning that $\Lambda$ is commensurable to $\Lambda^{\infty} \cap p^{-1}\left(W_{0}\right)$. Finally, $p_{\mid\left(\Lambda^{\infty} \cap p^{-1}\left(W_{0}\right)\right)^{\infty}}$ is a good model of $\Lambda^{\infty} \cap p^{-1}\left(W_{0}\right)$. Hence, $\Lambda$ is a Meyer subset. 
Proposition 7 Let $\Lambda \subset G$ be a uniform approximate lattice in a connected soluble Lie group. Then there is a uniform approximate lattice $\Lambda^{\prime}$ commensurable to $\Lambda$ such that $\left(\Lambda^{\prime}\right)^{\infty}$ is polycyclic.

Proof Let us first show that we can assume $G$ to be simply connected. Indeed, if $G$ is not simply connected we proceed as follows. Let $p: \tilde{G} \rightarrow G$ be a universal cover, then $p^{-1}(\Lambda)$ is a uniform approximate lattice in $\tilde{G}$. Suppose $p^{-1}(\Lambda)$ is commensurable to an approximate subgroup $\Lambda^{\prime}$ such that $\Lambda^{\prime}$ generates a polycyclic group. Then $p\left(\Lambda^{\prime}\right)$ is commensurable to $\Lambda$ and $p\left(\Lambda^{\prime}\right)$ generates a polycyclic group as well.

From now on $G$ is supposed simply connected. Let $N$ denote the nilpotent radical of $G, k \in \mathbb{N}$ and $\Xi \subset \Lambda^{k} \cap N$ be an approximate subgroup. First of all, let us show that $\Xi^{\infty}$ is finitely generated. Since $G$ is simply connected, $G$ does not contain any nontrivial compact subgroup. So $N$ does not contain any non-trivial compact subgroup, and thus $N$ is simply connected. Now $N$ is a connected simply connected nilpotent Lie group so it is the group of $\mathbb{R}$-points of a unipotent algebraic group (see [17, Theorem 4.1]) and $\Xi$ is a discrete approximate subgroup. Hence, $\Xi^{\infty}$ is finitely generated by Corollary 1.

The rest of the proof will rely on the following lemma that links finitely generated subgroups of connected simply connected nilpotent Lie groups to finite dimensional Q-Lie algebras.

Lemma 5 [17, Chapter IV] Let $\Gamma \subset N$ be a finitely generated group in a connected simply connected nilpotent Lie group. Then $\Gamma$ is torsion-free nilpotent, $\mathbb{Q} \log (\Gamma)$ is a finite dimensional $\mathbb{Q}$ Lie algebra and $\operatorname{dim}_{\mathbb{Q}}(\mathbb{Q} \log (\Gamma))=\operatorname{rank}(\Gamma)$.

Here the rank of $\Gamma$ is the dimension of its Malcev completion, i.e. the unique connected simply connected nilpotent Lie group that admits a lattice isomorphic to $\Gamma$, and $\log$ denotes the logarithm map from $N$ to its Lie algebra. Lemma 5 is a consequence of [17, Theorems 2.18, 2.12, 2.10 and 2.11].

The group $\Xi^{\infty}$ is finitely generated, torsion-free and nilpotent so it has finite rank. Among all approximate subgroups $\Xi$ commensurable to $\Lambda^{2} \cap N$ - note that $\Lambda^{2} \cap N$ is an approximate subgroup by Lemma 1 - such that there is $k \in \mathbb{N}$ satisfying $\Xi \subset \Lambda^{k} \cap N$, choose one with minimal rank. Let $\Xi$ denote this approximate subgroup and let $k$ be such that $\Xi \subset \Lambda^{k} \cap N$.

Take $\lambda \in \Lambda$. Note that by Lemma 1 and since

$$
\Lambda^{k} \cap N \subset \lambda\left(\Lambda^{k} \cap N\right) \lambda^{-1} \subset \Lambda^{k+2} \cap N
$$

the subsets $\Lambda^{k} \cap N, \lambda\left(\Lambda^{k} \cap N\right) \lambda^{-1}$ and $\Lambda^{k+2} \cap N$ are pairwise commensurable approximate subgroups. Therefore, $\Xi$ and $\lambda \Xi \lambda^{-1}$ are contained in and commensurable to $\Lambda^{k+2} \cap N$. So $\Xi^{2} \cap \lambda \Xi^{2} \lambda^{-1}$ is an approximate subgroup commensurable to $\Xi$ according to Lemma 2. But $\left(\Xi^{2} \cap \lambda \Xi^{2} \lambda^{-1}\right)^{\infty} \subset \Xi^{\infty}$ so these two subgroups have the same rank. As a consequence, $\left(\Xi^{2} \cap \lambda \Xi^{2} \lambda^{-1}\right)^{\infty}$ has finite index in both $\Xi^{\infty}$ and $\lambda \Xi^{\infty} \lambda^{-1}$. So the subgroups $\Xi^{\infty}$ and $\lambda \Xi^{\infty} \lambda^{-1}$ are commensurable. There is thus $n \in \mathbb{N}$ such that for all $\gamma \in \Xi^{\infty}$, we have $\gamma^{n} \in \Xi^{\infty} \cap \lambda \Xi^{\infty} \lambda^{-1}$. Therefore,

$$
n \log \left(\Xi^{\infty}\right) \subset \log \left(\lambda \Xi^{\infty} \lambda^{-1}\right) .
$$


Hence,

$$
\mathbb{Q} \log \left(\Xi^{\infty}\right)=\mathbb{Q} \log \left(\lambda \Xi^{\infty} \lambda^{-1}\right)
$$

We have thus proved that $\exp \left(\mathbb{Q} \log \left(\Xi^{\infty}\right)\right)$ is stable under conjugation by elements of $\Lambda^{\infty}$. Moreover, $\exp \left(\mathbb{Q} \log \left(\Xi^{\infty}\right)\right)$ is a group by the Baker-Campbell-Hausdorff formula (see [17, Chapter IV]) and any finitely generated subgroup in it has rank less than or equal to $\operatorname{dim}_{\mathbb{Q}}\left(\mathbb{Q} \log \left(\Xi^{\infty}\right)\right)=\operatorname{rank}\left(\Xi^{\infty}\right)$ according to Lemma 5 . Let $\Gamma$ denote the subgroup generated by $\Lambda^{k+2} \cap \exp \left(\mathbb{Q} \log \left(\Xi^{\infty}\right)\right)$. Since $\left(\Lambda^{k+2} \cap N\right)^{\infty}$ is nilpotent and finitely generated according to a combination of Lemma 1 and Corollary 1 , the subgroup $\Gamma$ is finitely generated as well. In addition, $\Gamma$ contains $\Xi^{\infty}$. So $\operatorname{rank}(\Gamma)=$ $\operatorname{rank}\left(\Xi^{\infty}\right)$ and $\Xi^{\infty}$ has finite index in $\Gamma$.

We know by [17, Theorem 2.12] that there exist free abelian subgroups $\Gamma_{1}, \Gamma_{2} \subset$ $\mathbb{Q} \log \left(\Xi^{\infty}\right)$ of $\operatorname{rank} \operatorname{dim}_{\mathbb{Q}}\left(\mathbb{Q} \log \left(\Xi^{\infty}\right)\right)$ such that

$$
\Gamma_{1} \subset \log \left(\Xi^{\infty}\right) \subset \log (\Gamma) \subset \Gamma_{2}
$$

This implies in particular that for all $\lambda \in \Lambda$ we have $\operatorname{Ad}(\lambda)\left(\Gamma_{1}\right) \subset \Gamma_{2}$ since $\lambda \Xi^{\infty} \lambda^{-1} \subset \Gamma$. According now to Proposition 4, there is an approximate subgroup $X \subset \operatorname{Ad}(\Lambda)^{4}$ commensurable to $\operatorname{Ad}(\Lambda)$ such that $\phi\left(\Gamma_{1}\right)=\Gamma_{1}$ for all $\phi \in X$. Define $\tilde{\Lambda}:=\operatorname{Ad}^{-1}(X)^{2} \cap \Lambda^{2}$. By Lemma 2 we find that $\tilde{\Lambda}$ is an approximate subgroup commensurable to $\Lambda$. And by construction $\operatorname{Ad}(\lambda)\left(\Gamma_{1}\right)=\Gamma_{1}$ for all $\lambda \in \tilde{\Lambda}$. Therefore, the subgroup $H$ of $\Xi^{\infty}$ generated by $\exp \left(\Gamma_{1}\right)$ has finite index in $\Xi^{\infty}$ and $H \cap \tilde{\Lambda}^{\infty}$ is normalised by $\tilde{\Lambda}$.

Consider $p: \tilde{\Lambda}^{\infty} \rightarrow \tilde{\Lambda}^{\infty} /\left(H \cap \tilde{\Lambda}^{\infty}\right)$ the canonical projection. We claim that $p\left(\tilde{\Lambda}^{2}\right) \cap Z\left(\tilde{\Lambda}^{\infty} /\left(H \cap \tilde{\Lambda}^{\infty}\right)\right)$ is commensurable to $p(\tilde{\Lambda})$. First, note that $\Xi$ is commensurable to $\Xi^{2} \cap H$ by Lemma $2, \Lambda^{8} \cap N$ is commensurable to $\Xi$ by Lemma 1 and $\tilde{\Lambda} \subset \Lambda^{2}$. Note also that $N$ contains all commutators of elements of $G$. So the set of commutators of elements of $p(\tilde{\Lambda})$ is contained in $p\left(\Lambda^{8} \cap N\right)$. But $p\left(\Lambda^{8} \cap N\right)$ is commensurable to $p\left(\Xi^{2} \cap H\right)=\{e\}$, and, hence, is finite. But $p\left(\tilde{\Lambda}^{\infty}\right)$ is finitely generated since $\tilde{\Lambda}$ is uniform approximate lattice in a connected Lie group ( see the proof of Proposition 3 or [2, Theorem 1.13]), and $p(\tilde{\Lambda})$ generates $p\left(\tilde{\Lambda}^{\infty}\right)$. We can therefore find a finite generating family $\Upsilon:=\left\{\gamma_{1}, \ldots, \gamma_{n}\right\} \subset p(\tilde{\Lambda})$. Define now

$$
\begin{aligned}
\theta_{\Upsilon}: p\left(\tilde{\Lambda}^{\infty}\right) & \rightarrow p\left(\tilde{\Lambda}^{\infty}\right)^{n} \\
\gamma & \mapsto\left(\left[\gamma_{1}, \gamma\right], \ldots,\left[\gamma_{n}, \gamma\right]\right),
\end{aligned}
$$

where $\left[\gamma_{i}, \gamma\right]:=\gamma_{i} \gamma \gamma_{i}^{-1} \gamma^{-1}$. Note now that for all $\gamma, \gamma^{\prime}$ in $p(\tilde{\Lambda})$ if $\theta_{\Upsilon}(\gamma)=\theta_{\Upsilon}\left(\gamma^{\prime}\right)$ then

$$
\gamma Z\left(\tilde{\Lambda}^{\infty} /\left(H \cap \tilde{\Lambda}^{\infty}\right)\right)=\gamma^{\prime} Z\left(\tilde{\Lambda}^{\infty} /\left(H \cap \tilde{\Lambda}^{\infty}\right)\right) .
$$

Indeed, for $i \in\{1, \ldots, n\}$ we have

$$
\gamma_{i} \gamma \gamma_{i}^{-1} \gamma^{-1}=\left[\gamma_{i}, \gamma\right]=\left[\gamma_{i}, \gamma^{\prime}\right]=\gamma_{i} \gamma^{\prime} \gamma_{i}^{-1} \gamma^{\prime-1}
$$


which implies

$$
\left(\gamma_{i} \gamma^{-1} \gamma_{i}^{-1}\right) \gamma_{i} \gamma^{\prime} \gamma_{i}^{-1} \gamma^{\prime-1}(\gamma)=\left[\gamma_{i}, \gamma^{-1} \gamma^{\prime}\right]=e
$$

So $\gamma^{-1} \gamma^{\prime}$ belongs to the centraliser of $\Upsilon$ i.e. belongs to the centre $Z\left(\tilde{\Lambda}^{\infty} /\left(H \cap \tilde{\Lambda}^{\infty}\right)\right)$ of $\tilde{\Lambda}^{\infty} /\left(H \cap \tilde{\Lambda}^{\infty}\right)$ because $\Upsilon$ is a generating family.

But $\theta_{\Upsilon}(p(\tilde{\Lambda}))$ is finite so there are $\gamma_{1}^{\prime}, \ldots, \gamma_{r}^{\prime} \in \tilde{\Lambda}^{\infty} /\left(H \cap \tilde{\Lambda}^{\infty}\right)$ such that $p(\tilde{\Lambda}) \subset$ $\bigcup \gamma_{i}^{\prime} Z\left(\tilde{\Lambda}^{\infty} /\left(H \cap \tilde{\Lambda}^{\infty}\right)\right)$. We thus have that $\tilde{\Lambda}$ is covered by finitely many left-cosets of $p^{-1}\left(Z\left(\tilde{\Lambda}^{\infty} /\left(H \cap \tilde{\Lambda}^{\infty}\right)\right)\right)$. According to Lemma 2 now, $\Lambda^{\prime}:=\tilde{\Lambda}^{2} \cap p^{-1}\left(Z\left(\tilde{\Lambda}^{\infty} /(H \cap\right.\right.$ $\left.\left.\tilde{\Lambda}^{\infty}\right)\right)$ ) is an approximate subgroup commensurable to $\tilde{\Lambda}$ and $\Lambda$.

Finally, $\Lambda^{\prime}$ is a uniform approximate lattice in $G$ as it is commensurable to $\Lambda$. So it generates a finitely generated subgroup (as above see the proof of Proposition 3 or [2, Theorem 1.13]). Moreover, $H \cap \tilde{\Lambda}^{\infty} \subset \Lambda^{\prime \infty}$ is a finitely generated torsion-free nilpotent normal subgroup such that $\Lambda^{\prime \infty} /\left(H \cap \tilde{\Lambda}^{\infty}\right)$ is abelian and finitely generated. Hence, $\Lambda^{\prime \infty}$ is polycyclic.

Proof of Theorem 3 Let $\Lambda \subset G$ be a uniform approximate lattice in a connected soluble Lie group. According to Proposition $7 \Lambda$ is commensurable to a uniform approximate lattice $\Lambda^{\prime}$ with $\Lambda^{\prime}$ polycyclic. By Proposition 6 the uniform approximate lattice $\Lambda^{\prime}$ is therefore a Meyer set. So $\Lambda$ is a Meyer set.

Acknowledgements I am deeply grateful to my supervisor, Emmanuel Breuillard, for his patient guidance and encouragements. I would also like to thank the anonymous referee for many helpful comments.

Data availabitlity Data sharing not applicable to this article as no datasets were generated or analysed during the current study.

\section{Declarations}

Conflict of interest The authors declare that they have no conflict of interest.

Open Access This article is licensed under a Creative Commons Attribution 4.0 International License, which permits use, sharing, adaptation, distribution and reproduction in any medium or format, as long as you give appropriate credit to the original author(s) and the source, provide a link to the Creative Commons licence, and indicate if changes were made. The images or other third party material in this article are included in the article's Creative Commons licence, unless indicated otherwise in a credit line to the material. If material is not included in the article's Creative Commons licence and your intended use is not permitted by statutory regulation or exceeds the permitted use, you will need to obtain permission directly from the copyright holder. To view a copy of this licence, visit http://creativecommons.org/licenses/by/4.0/.

\section{References}

1. Auslander, L.: On a Problem of Philip Hall. Ann. Math. 86(1), 112-116 (1967). http://www.jstor.org/ stable/1970362

2. Björklund, M., Hartnick, T.: Approximate lattices. Duke Math. J. 167(15), 2903-2964 (2018). https://doi.org/10.1215/00127094-2018-0028. https://doiorg.ezp.lib.cam.ac.uk/10.1215/001270942018-0028 
3. Björklund, M., Hartnick, T., Stulemeijer, T.: Borel density for approximate lattices. Forum Math. Sigma 7(e40), 27 (2019). https://doi.org/10.1017/fms.2019.39

4. Breuillard, E., Green, B., Tao, T.: The structure of approximate groups. Publ. Math. Inst. Hautes Études Sci. 116, 115-221 (2012). https://doi.org/10.1007/s10240-012-0043-9. https://doiorg.ezp.lib.cam.ac. uk/10.1007/s10240-012-0043-9

5. Carolino, P.K.: The Structure of Locally Compact Approximate Groups. ProQuest LLC, Ann Arbor, MI. Thesis (Ph.D.) University of California, Los Angeles (2015). http://gateway.proquest.com.ezp. lib.cam.ac.uk/openurl?url_ver=Z39.88-2004\&rft_val_fmt=info:ofi/fmt:kev:mtx:dissertation\&res_ dat=xri:pqm\&rft_dat=xri:pqdiss:3725297

6. Fish, A.: Extensions of Schreiber's theorem on discrete approximate subgroups in $\mathbb{R}^{d}$. J. Éc. Polytech. Math. 6, 149-162 (2019). https://doi.org/10.5802/jep.90

7. Freı̌man, G.A.: Foundations of a structural theory of set addition. American Mathematical Society, Providence, R.I. (1973) (Translated from the Russian, Translations of Mathematical Monographs, Vol 37)

8. Fried, D., Goldman, W.M.: Three-dimensional affine crystallographic groups. Adv. Math. 47(1), 1-49 (1983). https://doi.org/10.1016/0001-8708(83)90053-1. https://doi-org.ezp.lib.cam.ac.uk/10. 1016/0001-8708(83)90053-1

9. Helfgott, H.A.: Growth in groups: ideas and perspectives. Bull. Amr. Math. Soc. (N.S.) 52(3), 357413 (2015). https://doi.org/10.1090/S0273-0979-2015-01475-8. https://doi-org.ezp.lib.cam.ac.uk/10. 1090/S0273-0979-2015-01475-8

10. Hrushovski, E.: Stable group theory and approximate subgroups. J. Am. Math. Soc. 25(1), 189-243 (2012). https://doi.org/10.1090/S0894-0347-2011-00708-X

11. Macbeath, A.M., Świerczkowski, S.: On the set of generators of a subgroup. Nederl. Akad. Wetensch. Proc. Ser. A 62 Indag. Math. 21, 280-281 (1959)

12. Machado, S.: Approximate lattices and Meyer sets in nilpotent Lie groups. Discrete Anal. pp. Paper No. 1, 18 (2020). https://doi.org/10.19086/da.11886. https://doi-org.ezp.lib.cam.ac.uk/10.19086/da. 11886

13. Machado, S.: Good models, infinite approximate subgroups and approximate lattices (2020). arXiv:2011.01829

14. Massicot, J.C., Wagner, F.O.: Approximate subgroups. J. Éc. Polytech. Math. 2, 55-64 (2015). https:// doi.org/10.5802/jep. 17

15. Meyer, Y.: Algebraic numbers and harmonic analysis, vol. 2. Elsevier, Amsterdam (1972)

16. Pyber, L., Szabó, E.: Growth in finite simple groups of Lie type. J. Am. Math. Soc. 29(1), 95146(2016). https://doi.org/10.1090/S0894-0347-2014-00821-3. https://doi-org.ezp.lib.cam.ac.uk/10. 1090/S0894-0347-2014-00821-3

17. Raghunathan, M.S.: Discrete subgroups of Lie groups. Ergebnisse der Mathematik und ihrer Grenzgebiete, Band 68. Springer, New York, Heidelberg (1972)

18. Ruzsa, I.Z.: Arithmetical progressions and the number of sums. Period. Math. Hung. 25(1), 105-111 (1992). https://doi.org/10.1007/BF02454387. https://doi-org.ezp.lib.cam.ac.uk/10.1007/BF02454387

19. Schreiber, J.P.: Approximations diophantiennes et problemes additifs dans les groupes abéliens localement compacts. Bull. Soc. Math. Fr. 101, 297-332 (1973)

20. Springer, T.A.: Linear Algebraic Groups. Springer, New York (2010)

21. Tao, T.: Product set estimates for non-commutative groups. Combinatorica 28(5), 547-594 (2008). https://doi.org/10.1007/s00493-008-2271-7. https://doi-org.ezp.lib.cam.ac.uk/10.1007/s00493-0082271-7

22. Tointon, M.C.H.: Introduction to Approximate Groups, London Mathematical Society Student Texts. Cambridge University Press, Cambridge (2019)

23. Whitney, H.: Elementary structure of real algebraic varieties. Ann. Math. 2(66), 545-556 (1957). https://doi.org/10.2307/1969908

24. Witte, D.: Zero-entropy affine maps on homogeneous spaces. Am. J. Math. 109(5), 927-961 (1987). https://doi.org/10.2307/2374495

25. Witte, D.: Superrigidity of lattices in solvable Lie groups. Invent. Math. 122(1), 147-193 (1995). https://doi.org/10.1007/BF01231442

Publisher's Note Springer Nature remains neutral with regard to jurisdictional claims in published maps and institutional affiliations. 